\title{
Mitochondrial iron transport and homeostasis in plants
}

\section{Anshika Jain and Erin L. Connolly*}

Department of Biological Sciences, University of South Carolina, Columbia, SC, USA

\author{
Edited by: \\ Gianpiero Vigani, Università degli \\ Studi di Milano, Italy \\ Reviewed by: \\ Elsbeth L. Walker, University of \\ Massachusetts, Amherst, USA \\ Michael A. Grusak, United States \\ Department of Agriculture-Agricultural \\ Research Service Children's Nutrition \\ Research Center, USA \\ *Correspondence: \\ Erin L. Connolly, University of South \\ Carolina, 715 Sumter Street, \\ Columbia, SC 29208, USA \\ e-mail:erinc@biol.sc.edu
}

\begin{abstract}
Iron $(\mathrm{Fe})$ is an essential nutrient for plants and although the mechanisms controlling iron uptake from the soil are relatively well understood, comparatively little is known about subcellular trafficking of iron in plant cells. Mitochondria represent a significant iron sink within cells, as iron is required for the proper functioning of respiratory chain protein complexes. Mitochondria are a site of $\mathrm{Fe}-\mathrm{S}$ cluster synthesis, and possibly heme synthesis as well. Here we review recent insights into the molecular mechanisms controlling mitochondrial iron transport and homeostasis. We focus on the recent identification of a mitochondrial iron uptake transporter in rice and a possible role for metalloreductases in iron uptake by mitochondria. In addition, we highlight recent advances in mitochondrial iron homeostasis with an emphasis on the roles of frataxin and ferritin in iron trafficking and storage within mitochondria.
\end{abstract}

Keywords: iron, mitochondria, plant, iron transporter, frataxin, ferritin

\section{INTRODUCTION}

Iron is an essential micronutrient for virtually all organisms, including plants. Indeed, photosynthetic organisms are distinguished by the high iron requirement of photosynthetic complexes. Although iron is generally quite abundant in the soil, it has a low bioavailability in aerobic environments at neutral to basic $\mathrm{pH}$ and as a result, approximately $30 \%$ of the world's soils are considered iron-limiting for plant growth. Iron deficiency represents an enormous problem in human populations as well, with approximately two billion people afflicted (Wu et al., 2002). Plant foods (especially staples like rice, maize, and wheat) tend to be poor sources of dietary iron and thus significant interest surrounds efforts to develop crop varieties with elevated levels of bioavailable iron.

Despite its importance, iron can be toxic when it accumulates to high levels within cells. It catalyzes the formation of hydroxyl radicals that can damage cellular components like DNA and proteins (Halliwell etal., 1992). Thus iron metabolism is carefully regulated to ensure adequate supply of iron while avoiding toxicity associated with its over-accumulation. Organelles like chloroplasts and mitochondria are thought to play a central role in the cellular iron economy of a plant cell. This is because iron serves as an essential cofactor for many enzymes involved in the electron transport chain in mitochondria and in the photosynthetic complexes found in chloroplasts. Indeed, recent work has shown that iron deficiency results in significant changes in the structure and function of mitochondria (Vigani et al., 2009; Vigani and Zocchi, 2009). PS1-LHC1 supercomplexes also exhibit structural and functional alterations under iron limited conditions (Yadavalli et al., 2012). Thus, iron deficiency affects respiratory and photosynthetic output. However, excessive iron exacerbates the generation of reactive oxygen species (ROS) in these redox centers, which can have exceedingly deleterious effects on cells. Thus, mitochondrial and chloroplast iron metabolism are of particular importance to cellular iron homeostasis (Nouet et al., 2011; Vigani et al., 2013).
The chloroplasts and mitochondria are unique organelles in that they are thought to have evolved via endosymbiosis. As a result, both organelles are surrounded by two membranes; the outer membrane resembles eukaryotic membranes while the inner resembles prokaryotic membranes. Thus, it follows that these two organelles may utilize prokaryotic and/or eukaryotic type iron transport systems (Shimoni-Shor et al., 2010). It is usually assumed that Fe may pass freely across the outer membrane of both organelles via porins. It is also important to note that there is little known about the speciation of cytosolic Fe although it is assumed that there is very little free Fe present in the cytosol (Hider and Kong, 2013). Thus, the Fe species that are available for transport into subcellular compartments are unclear at this time.

A variety of proteins are known to be involved in the maintenance of mitochondrial iron homeostasis (Figure 1). The recent discovery and characterization of rice MIT (mitochondrial iron transporter), which is involved in iron uptake by mitochondria, and the mitochondrial iron chaperone, frataxin $(\mathrm{FH})$ has demonstrated the significance of mitochondrial iron uptake and trafficking/distribution to plant growth and development (Busi et al., 2006; Bashir et al., 2011b; Maliandi et al., 2011; Vigani, 2012). Other key players responsible for maintaining mitochondrial iron homeostasis are the iron-sulfur cluster (ISC) synthesis machinery, which accepts iron from $\mathrm{FH}$ and mediates the synthesis of $\mathrm{Fe}-\mathrm{S}$ clusters to serve as cofactors for various proteins in the mitochondria and cytosol (Lill et al., 2012). It is thought that mitochondria also contain the iron storage protein ferritin, which serves to store iron and protect against Fe-catalyzed ROS production (Briat et al., 2010). Mitochondria also supply iron and sulfur to the cytoplasmic iron-sulfur cluster assembly machinery (CIA; Balk and Pilon, 2011). While the sulfur scaffold is likely exported to the cytosol via an ABC transporter, ATM3 (Bernard et al., 2009), putative transporters required for iron efflux are still unknown. The recent discovery of a mitochondrial iron exporter (MIE) in mice (ATP-binding cassette B8, ABCB8) and its role in CIA-mediated $\mathrm{Fe}-\mathrm{S}$ synthesis has provided new insight into understanding of 


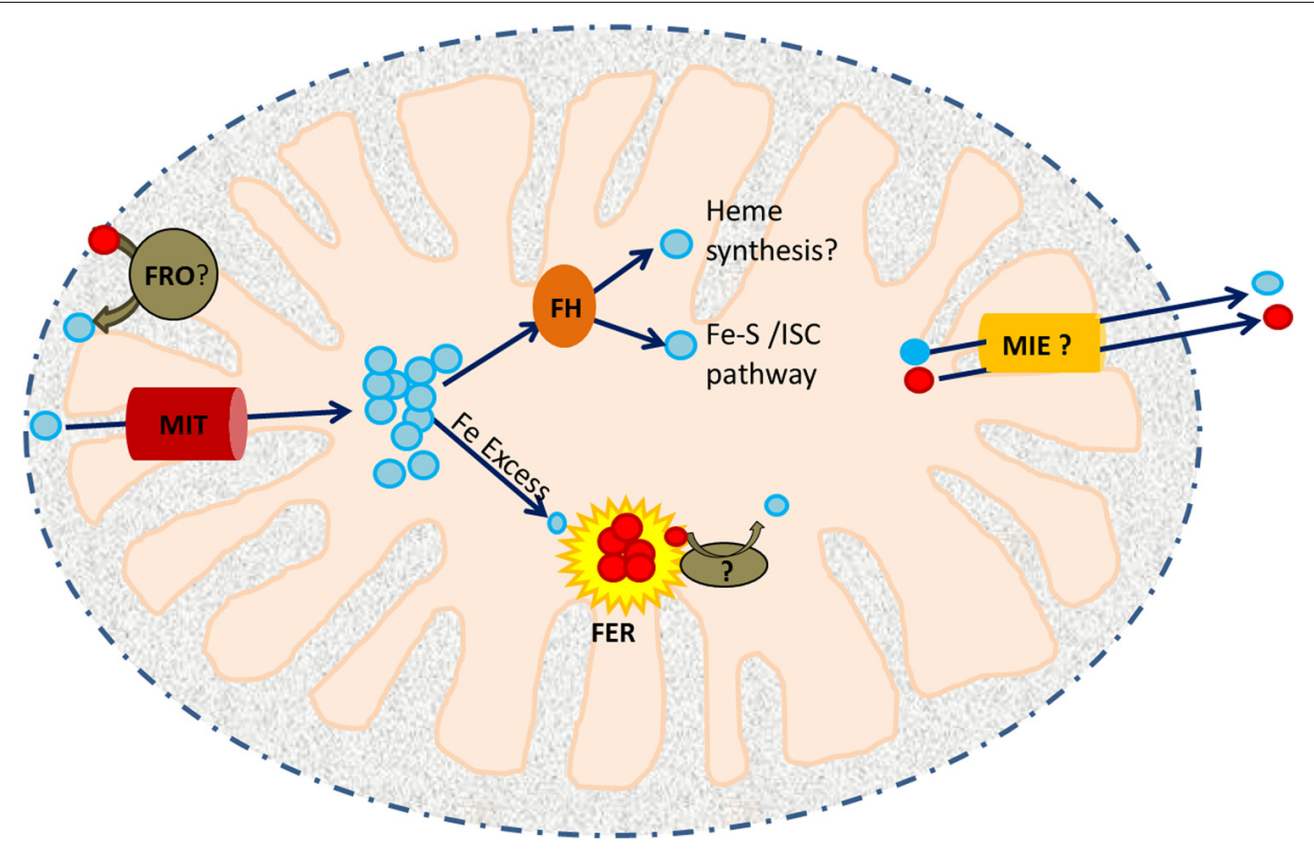

FIGURE 1 | A working model of iron trafficking and utilization in plant mitochondria. Cytosolic $\mathrm{Fe}^{3+}$ (red circles) may be reduced to $\mathrm{Fe}^{2+}$ (blue circles) by a member of the ferric reductase oxidase (FRO) family within the inter-membrane space (IMS). Ferrous iron is then translocated across the inner membrane by MIT. In the mitochondrial matrix, it is received by an iron chaperone, frataxin (FH). FH distributes this Fe to ISC assembly proteins and possibly to the heme biosynthetic machinery. Excess $\mathrm{Fe}^{2+}$ is stored in FER4. Iron released from FER4 upon Fe deficiency may require the activity of another reductase prior to its utilization/remobilization. Mitochondrial iron exporters (MIEs) are postulated to function in mitochondrial iron export for delivery of iron to $\mathrm{ClA}$. intracellular iron homeostasis (Ichikawa et al., 2012) and may facilitate the identification of MIE proteins in other species. Finally, a recent report showed that the YSL4 and YSL6 (yellow stripelike) transporters are involved in iron release from chloroplasts of Arabidopsis, suggesting a possible role for other members of the YSL family in mitochondrial iron efflux (Divol et al., 2013). In this review, we discuss in detail the roles of the iron transporter (MIT), metalloreductases, FH and ferritin in mitochondrial iron homeostasis.

\section{MITOCHONDRIAL IRON TRANSPORTERS}

Mitochondrial iron transporters are conserved proteins that belong to the mitochondrial carrier family (MCF; Wiesenberger et al., 1991; Metzendorf et al., 2009; Bashir et al., 2011a,b). This family consists of small $(\sim 30 \mathrm{kDa})$ proteins that localize to the mitochondrial inner membrane and are involved in solute transport (e.g., keto acids, nucleotides, amino acids, etc.) into the mitochondrial matrix (Kunji and Robinson, 2006). MCF proteins were first characterized in yeast and their crystal structure shows the presence of a tripartite structure with a total of six transmembrane helices. Amino acid residues responsible for substrate recognition are found in helices II, IV and VI.

The first set of mitochondrial iron transporters (MRS3 and MRS4 for mitochondrial RNA splicing) were discovered in yeast as multicopy suppressors of the mrs 2 phenotype (Waldherr et al., 1993) and subsequently shown to function in Fe import (Foury and Roganti, 2002). Mitochondrial iron transporters (named Mitoferrins) have since been identified and characterized in zebrafish, humans, and Drosophila (Shaw et al., 2006; Metzendorf et al., 2009; Paradkar et al., 2009). Later, a mitochondrial iron transporter was identified in rice and named MIT (Bashir et al., 2011b). Similar to zebrafish mitoferrin, rice $M I T$ is able to rescue the poor growth phenotype of the yeast $m r s 3 / 4$ mutant under iron deficiency, indicating the functional similarity of these proteins. MIT is an essential gene as the mit knockout mutant shows an embryo lethal phenotype. mit knockdown mutants exhibit a slow growth phenotype, reduced chlorophyll concentration and poor seed yield. In addition, these mutants show reduced mitochondrial iron concentration while total iron concentration is elevated, indicating that iron is mislocalized in mit loss-of-function lines (Bashir et al., 2011b). In the absence of MIT, the gene encoding vacuolar iron transporter1 (VIT1) is upregulated, suggesting that excess cytosolic iron may be directed toward vacuoles. MIT plays an important role in seed development and its expression level is positively regulated by iron availability. MIT is expressed throughout development, consistent with the idea that it is essential for mitochondrial iron metabolism.

Previous studies conducted in yeast and mammals have demonstrated an adverse effect of loss of mitochondrial iron transport on heme and Fe-S cluster synthesis (Zhang et al., 2005; Shaw et al., 2006; Zhang et al., 2006). In rice, partial loss of MIT results in decreased total and mitochondrial aconitase activity, indicating that the effect on Fe-S cluster synthesis affects not only mitochondrial $\mathrm{Fe}-\mathrm{S}$ proteins but also cytosolic Fe-S cluster proteins. However, the role of MIT in heme synthesis has yet to be determined. Interestingly, the fact that mit loss-of-function 
lines show altered chlorophyll concentration and altered ferritin expression supports the idea of cross-talk between mitochondrial and chloroplastic iron homeostasis.

Yeast MRS3/4 are thought to serve as high affinity ferrous ion transporters which are essential in the absence of other low affinity mitochondrial iron transporters (Froschauer et al., 2009). Thus, low affinity iron uptake systems may be present at the plant mitochondrial membrane. The recent discovery of siderophore (2,5-DHBA)-mediated iron delivery to mammalian mitochondria has introduced the possibility of such an alternative pathway for iron transport into the mitochondria (Devireddy et al., 2010). 2,5DHBA is synthesized by a short chain dehydrogenase/reductase family member (BDH2). BLAST searches of the Arabidopsis and rice genomes indicate that these genomes code for 3 and $13 \mathrm{BDH} 2$ homologs respectively. Characterization of these homologs may give interesting insights into the mitochondrial iron trafficking pathways in plants.

\section{PUTATIVE MITOCHONDRIAL FERRIC REDUCTASES}

The recent characterization of Arabidopsis FRO7 (ferric reductase oxidase 7), a chloroplast-localized member of the FRO family, demonstrated that this protein functions to reduce ferric iron to ferrous iron at the surface of the chloroplast for subsequent uptake into the organelle (Jeong et al., 2008). A similar hypothesis has been suggested for mitochondria based on the predicted localization of FRO3 and FRO 8 to mitochondria (Jeong and Connolly, 2009). Whereas FRO3-GUS promoter lines show expression throughout seedlings with highest expression in vasculature, expression of FRO8 is restricted to shoots during senescence (Mukherjee et al., 2006; Jeong and Connolly, 2009). This suggests that the two mitochondrial FROs may be involved in reducing $\mathrm{Fe}^{3+}$ at different stages of development. FRO3 has been widely used as an iron deficiency marker (Mukherjee et al., 2006). Nevertheless, the exact role of FRO3 in planta remains elusive. It is important to note that although mitochondrial homologs of FRO3 have been identified in other organisms (FRE5 in yeast) there is no evidence to date that these proteins function in mitochondrial iron metabolism (Jeong and Connolly, 2009).

Rice possesses only two FRO family members, OsFRO1 and OsFRO2, neither of which has been shown to localize to mitochondria (Victoria Fde et al., 2012; Vigani, 2012). Thus, iron uptake by mitochondria of grass species such as rice may differ from non-grass species, like Arabidopsis. It is possible that iron uptake by rice mitochondria utilizes a non-reductive iron uptake pathway and/or the rice genome may encode other types of reductases capable of reducing iron. In the future, it will be critical to determine the redox state of iron transported across the outer and inner membranes of the mitochondria.

\section{FRATAXIN}

Mitochondria are known to facilitate two major iron utilization pathways in the cell: heme synthesis and Fe-S cluster biogenesis. It has been suggested that the mitochondrial compartment contains micromolar concentrations of chelatable iron (Petrat et al., 2001). Maintaining this iron in a soluble and non-toxic form presents a challenge given the alkaline $\mathrm{pH}$ and the continuous production of ROS within the mitochondrial matrix under normal conditions (Park et al., 2002). Thus, the existence of mitochondrial iron chaperones and chelators was postulated (Flatmark and Romslo, 1975). Mitochondrial ferritin and FH have been implicated in iron storage and control of iron homeostasis in the mitochondrial matrix (Babcock et al., 1997; Corsi et al., 2002).

Frataxin is a conserved mitochondrial protein found in bacteria, yeast, mammals, and plants (Busi et al., 2006). FH was first identified in humans, where its deficiency was reported to cause an autosomal recessive cardio-neurodegenerative disease known as Friedreich's ataxia (Campuzano et al., 1996). Functional studies in yeast revealed the role of $\mathrm{FH}$ (mYfhlp) in mitochondrial iron homeostasis (Babcock et al., 1997). Loss of yeast mYfh1p results in impaired iron export from the mitochondria. This is primarily due to the accumulation of iron as amorphous ferric phosphate nanoparticles, which are unavailable for physiological purposes (Lesuisse et al., 2003). Thus, although the $y f h 1$ mutant exhibits iron overload, it suffers from iron deficiency and thus upregulates the iron uptake machinery (Santos et al., 2010). Lack of FH also results in iron-induced oxidative damage of mtDNA and reduced activity of mitochondrial Fe-S cluster proteins which thus affects respiration (Foury, 1999). FH has been reported to bind to the ISC assembly complex suggesting its importance in Fe-S cluster biogenesis (Gerber et al., 2003). It directly interacts with a scaffold protein, Isu (iron-sulfur cluster $\mathrm{U}$ ) in an iron-dependent manner and facilitates the transfer of iron to Isu during $\mathrm{Fe}-\mathrm{S}$ cluster assembly. Because of the capacity of FH to bind iron and transfer it to Isu via a direct protein-protein interaction, $\mathrm{FH}$ is considered a mitochondrial iron chaperone (Philpott, 2012).

The first $\mathrm{FH}$ homolog identified in a photosynthetic organism was Arabidopsis AtFH (Busi et al., 2004); AtFH functionally complements the yeast FH mutant. AtFH is essential as loss-offunction mutants exhibit an embryo lethal phenotype (Vazzola et al., 2007). The knock-down mutant shows elevated levels of iron and ROS in the mitochondrial compartment (Martin et al., 2009). The oxidative stress observed in atfh mutants is accompanied by an increase in nitric oxide (NO) production. NO, a potent antioxidant, protects the cell by directly scavenging peroxide radicals (Beligni and Lamattina, 1999) and by inducing the expression of ferritin genes (FER1 and FER4) to sequester free iron (Murgia et al., 2002; Martin et al., 2009).

Like its yeast ortholog, AtFH also functions as a mitochondrial iron chaperone. atfh mutants show reduced activity of two Fe-S cluster containing enzymes, mitochondrial aconitase and succinate dehydrogenase, while the activity of malate dehydrogenase (a non-Fe-S containing enzyme) is not altered. This indicates that $\mathrm{FH}$ likely plays a role in $\mathrm{Fe}-\mathrm{S}$ cluster biogenesis and/or assembly of the Fe-S moiety with mitochondrial proteins in Arabidopsis. Indeed, it was shown that AtFH plays an instrumental role in $\mathrm{Fe}-\mathrm{S}$ cluster biogenesis in plant mitochondria (Turowski et al., 2012). AtFH interacts with a cysteine desulfurase, AtNfs1m (which is known to supply $\mathrm{S}$ for the biogenesis of Fe-S clusters), and modulates its kinetic properties. AtNfs1m exhibits a 50-fold increase in its cysteine desulfurase activity in the presence of AtFH (Turowski et al., 2012). This interaction thus links the accumulation of iron (bound to $\mathrm{FH}$ ) with $\mathrm{Fe}-\mathrm{S}$ cluster production in a mitochondrion.

In animal systems, FH appears to be involved in the biogenesis of heme-containing proteins. FH was shown to interact 
with and deliver iron to ferrochelatase (FC) in the last step of heme synthesis in human mitochondria (Yoon and Cowan, 2004). Reduced FH expression in human cells results in reduced levels of heme-a and reduced cytochrome c oxidase activity (Napoli et al., 2007). In plants, however, there is no strong evidence to support the presence of FC in mitochondria. In fact, studies in various families of plants have clearly demonstrated the exclusive localization of FC to plastids (Cornah et al., 2002; Masuda et al., 2003). Therefore, it is possible that heme synthesis in plants occurs exclusively in plastids, some of which is then exported to the cytosol and mitochondria (van Lis et al., 2005; Tanaka and Tanaka, 2007; Mochizuki et al., 2010). Despite this, AtFH deficient plants show a decrease in total heme content, an altered expression of genes (FC2, HEMA1, HEMA2, GSA1, GSA2, HEMB2, HEMF2) which are involved in the heme biosynthetic pathway and a reduction in the activity of mitochondrial catalase, which is a heme-containing protein. Reduced catalase activity in atfh is rescued via supplementation with exogenous hemin (Maliandi et al., 2011). Taken together, these data indicate that in plants, FH plays important roles in protection against oxidative stress and in the biogenesis of $\mathrm{Fe}-\mathrm{S}$ cluster and heme-containing proteins (Maliandi et al., 2011).

\section{MITOCHONDRIAL FERRITIN}

Metal homeostasis in plants is accomplished via a set of elegantly regulated mechanisms that control various aspects of iron metabolism (including uptake, efflux, chelation, and storage). Ferritins are clearly essential to overall iron homeostasis as they function in iron sequestration and thus serve to prevent oxidative damage (Zhao et al., 2002; Arosio et al., 2009; Ravet et al., 2009). Ferritins are localized to both chloroplasts and mitochondria, two major sites for ROS production. Plant ferritins are conserved proteins that oligomerize to form a hollow sphere. They exhibit ferroxidase activity and oxidize $\mathrm{Fe}^{2+}$ and store it within the ferritin core in the form of hydrous ferric oxides along with phosphates (Arosio et al., 2009). Ferritin can accommodate 2,000-4,000 $\mathrm{Fe}^{3+}$ atoms per ferritin molecule (Carrondo, 2003). The molecular mechanism underlying the release of iron from ferritins is not very well understood. In vitro studies in animals suggest that release of Fe requires iron chelators or reducing agents. In contrast, in vivo studies in animals have demonstrated the release of Fe by proteolytic degradation of ferritin protein (Briat et al., 2010). To date, the process is not described in plant systems.

Plant ferritins are primarily localized to plastids, as opposed to animal ferritins which are usually cytoplasmic. Mitochondrial localization of ferritins was first reported in mammals (Levi et al.,

\section{REFERENCES}

Arosio, P., Ingrassia, R., and Cavadini, P. (2009). Ferritins: a family of molecules for iron storage, antioxidation, and more. Biochim. Biophys. Acta 7, 589-599. doi: 10.1016/j.bbagen.2008.09.004

Babcock, M., De Silva, D., Oaks, R., Davis-Kaplan, S., Jiralerspong, S., Montermini, L., et al. (1997). Regulation of mitochondrial iron accumulation by Yfhlp,

2001). Subsequently, mitochondrial ferritins were also identified in plants (Zancani et al., 2004; Tarantino et al., 2010a). Arabidopsis possesses four ferritin (FER1-4) proteins, all of which are known to be localized to chloroplasts. Ferritin4 (AtFER4) is unique in that it contains dual targeting signals and is therefore found in mitochondria as well as chloroplasts (Tarantino et al., 2010a). This protein is detected in mitochondria in the aerial portion of plants only after exposure to excess iron. Although the atfer 4 mutant does not exhibit any severe phenotypes, callus cultures prepared from the atfer 4 mutant show reduced cell and vacuole size, damaged plasma membranes, accumulation of $\mathrm{H}_{2} \mathrm{O}_{2}$, higher cell death and reduction of $\mathrm{O}_{2}$ consumption, in addition to elevated cellular and mitochondrial iron concentrations (Tarantino et al., 2010b).

Loss of AtFER4 also results in increased FRO3 expression both in control as well as excess Fe conditions. This suggests that loss of AtFER4 triggers sensing of mitochondrial iron deficiency despite the fact that mitochondrial iron levels are elevated in atfer4. This may also result in damage to electron transport chain components, which is consistent with the diminished $\mathrm{O}_{2}$ consumption rate of atfer 4 mutants. These observations indicate that although AtFER4 is responsible for proper cellular iron homeostasis and subcellular iron trafficking, it is dispensable for protection against the oxidative stress in photosynthetic tissue (Tarantino et al., 2010a).

\section{CONCLUSION}

Recent studies have begun to shed light on the machinery involved in mitochondrial iron uptake, storage, and trafficking/utilization. In particular, studies of mitochondrial iron transporters, chaperones, and storage proteins have set the stage for future investigations in this area (see Figure 1). Such studies will be critical to efforts to understand both organellar iron homeostasis and the mechanisms employed by plants to coordinate and prioritize Fe utilization by the various iron containing compartments of the cell. These studies will contribute to the development of a comprehensive understanding of iron homeostasis in plants, which should enable efforts to develop crop varieties with improved tolerance of growth on iron-limited soils and elevated levels of bioavailable iron in support of improved sustainability in agriculture and reductions in the incidence of iron deficiency in humans, respectively.

\section{ACKNOWLEDGMENT}

The authors are grateful to Grandon Wilson for critical reading of the manuscript and gratefully acknowledge support from the US NSF (IOS 0919739).

Signal. Behav. 6, 1591-1593. doi: 10.4161/psb.6.10.17132

Bashir, K., Ishimaru, Y., Shimo, H., Nagasaka, S., Fujimoto, M., Takanashi, H., et al. (2011b). The rice mitochondrial iron transporter is essential for plant growth. Nat. Commun. 2, 322 . doi: 10.1038/ncomms 1326

Beligni, M., and Lamattina, L. (1999). Is nitric oxide toxic or protective? Trends Plant Sci. 4, 299-300. doi: $\quad 10.1016 / S 1360-1385(99) 01$ 451-X

Bernard, D. G., Cheng, Y., Zhao, Y., and Balk, J. (2009). An allelic mutant series of ATM3 reveals its key role in the biogenesis of cytosolic iron-sulfur proteins in Arabidopsis. Plant Physiol. 151, 590-602. doi: 10.1104/pp.109.143651

Briat, J.-F., Duc, C., Ravet, K., and Gaymard, F. (2010). Ferritins and iron storage in plants. Biochim. 
Biophys. Acta 1800, 806-814. doi: 10.1016/j.bbagen.2009.12.003

Busi, M. V., Maliandi, M. V., Valdez, H., Clemente, M., Zabaleta, E. J., Araya, A., et al. (2006). Deficiency of Arabidopsis thaliana frataxin alters activity of mitochondrial $\mathrm{Fe}-\mathrm{S}$ proteins and induces oxidative stress. Plant J. 48, 873-882. doi: $10.1111 /$ j.1365313X.2006.02923.x

Busi, M. V., Zabaleta, E. J., Araya, A., and Gomez-Casati, D. F. (2004). Functional and molecular characterization of the frataxin homolog from Arabidopsis thaliana. FEBS Lett. 576, 141-144. doi: 10.1016/j.febslet.2004.09.003

Campuzano, V., Montermini, L., Molto, M. D., Pianese, L., Cossee, M., Cavalcanti, F., et al. (1996). Friedreich's ataxia: autosomal recessive disease caused by an intronic GAA triplet repeat expansion. Science 271, 1423-1427. doi: 10.1126/science.271.5254.1423

Carrondo, M. A. (2003). Ferritins, iron uptake and storage from the bacterioferritin viewpoint. EMBO J. 22, 1959-1968. doi: 10.1093/ emboj/cdg215

Cornah, J. E., Roper, J. M., Pal Singh, D., and Smith, A. G. (2002). Measurement of ferrochelatase activity using a novel assay suggests that plastids are the major site of haem biosynthesis in both photosynthetic and nonphotosynthetic cells of pea (Pisum sativum L.). Biochem. J. 362, 423-432.

Corsi, B., Cozzi, A., Arosio, P., Drysdale, J., Santambrogio, P., Campanella, A., et al. (2002). Human mitochondrial ferritin expressed in HeLa cells incorporates iron and affects cellular iron metabolism. J. Biol. Chem. 277, 22430-22437. doi: 10.1074/jbc.M105372200

Devireddy, L. R., Hart, D. O., Goetz, D. H., and Green, M. R. (2010). A mammalian siderophore synthesized by an enzyme with a bacterial homolog involved in enterobactin production. Cell 141, 1006-1017. doi: 10.1016/j.cell.2010.04.040

Divol, F., Couch, D., Conejero, G., Roschzttardtz, H., Mari, S., and Curie, C. (2013). The Arabidopsis YELLOW STRIPE LIKE4 and 6 transporters control iron release from the chloroplast. Plant Cell 25, 1040-1055. doi: $10.1105 /$ tpc.112.107672

Flatmark, T., and Romslo, I. (1975). Energy-dependent accumulation of iron by isolated rat liver mitochondria. Requirement of reducing equivalents and evidence for a unidirectional flux of $\mathrm{Fe}$ (II) across the inner membrane. J. Biol. Chem. 250, 6433-6438.
Foury, F. (1999). Low iron concentration and aconitase deficiency in a yeast frataxin homologue deficient strain. FEBS Lett. 456, 281284. doi: 10.1016/S0014-5793(99)0 0961-8

Foury, F., and Roganti, T. (2002). Deletion of the mitochondrial carrier genes MRS3 and MRS4 suppresses mitochondrial iron accumulation in a yeast frataxin-deficient strain. $J$. Biol. Chem. 277, 24475-24483. doi: 10.1074/jbc.M111789200

Froschauer, E. M., Schweyen, R. J., and Wiesenberger, G. (2009). The yeast mitochondrial carrier proteins Mrs3p/Mrs4p mediate iron transport across the inner mitochondrial membrane. Biochim. Biophys. Acta 1788, 1044-1050. doi: 10.1016/j. bbamem.2009.03.004

Gerber, J., Muhlenhoff, U., and Lill, R. (2003). An interaction between frataxin and Isul/Nfs1 that is crucial for $\mathrm{Fe} / \mathrm{S}$ cluster synthesis on Isu1. EMBO Rep. 4, 906-911. doi: 10.1038/sj.embor.embor918

Halliwell, B., Gutteridge, J. M., and Cross, C. E. (1992). Free radicals, antioxidants, and human disease: where are we now? J. Lab. Clin. Med. 119, 598-620.

Hider, R. C., and Kong, X. (2013). Iron speciation in the cytosol: an overview. Dalton Trans. 42, 3220-3229. doi $10.1039 / \mathrm{c} 2 \mathrm{dt} 32149 \mathrm{a}$

Ichikawa, Y., Bayeva, M., Ghanefar, M., Potini, V., Sun, L., Mutharasan, R. K., et al. (2012). Disruption of ATP-binding cassette B8 in mice leads to cardiomyopathy through a decrease in mitochondrial iron export. Proc. Natl. Acad. Sci. U.S.A. 109, 4152-4157. doi: 10.1073/pnas. 1119338109

Jeong, J., Cohu, C., Kerkeb, L., Pilon, M., Connolly, E. L., and Guerinot, M. L. (2008). Chloroplast Fe(III) chelate reductase activity is essential for seedling viability under iron limiting conditions. Proc. Natl. Acad. Sci. U.S.A. 105, 10619-10624. doi: 10.1073/pnas.0708367105

Jeong, J., and Connolly, E. L. (2009). Iron uptake mechanisms in plants: functions of the FRO family of ferric reductases. Plant Sci. 176, 709-714.

Kunji, E. R., and Robinson, A. J. (2006). The conserved substrate binding site of mitochondrial carriers. Biochim. Biophys. Acta 1757, 1237-1248. doi: 10.1016/j.bbabio.2006.03.021

Lesuisse, E., Santos, R., Matzanke, B. F., Knight, S. A., Camadro, J. M., and Dancis, A. (2003). Iron use for haeme synthesis is under control of the yeast frataxin homologue (Yfh1). Hum. Mol. Genet. 12, 879-889.
Levi, S., Corsi, B., Bosisio, M., Invernizzi, R., Volz, A., Sanford, D., et al. (2001). A human mitochondrial ferritin encoded by an intronless gene. J. Biol. Chem. 276, 24437-24440. doi: 10.1074/jbc.C100141200

Lill, R., Hoffmann, B., Molik, S., Pierik, A. J., Rietzschel, N., Stehling, O., et al. (2012). The role of mitochondria in cellular iron-sulfur protein biogenesis and iron metabolism. Biochim. Biophys. Acta 1823, 1491-1508. doi: 10.1016/j.bbamcr.2012.05.009

Maliandi, M. V., Busi, M. V., Turowski, V. R., Leaden, L., Araya, A., and Gomez-Casati, D. F. (2011). The mitochondrial protein frataxin is essential for heme biosynthesis in plants. FEBS J. 278, 470-481. doi: 10.1111/j.1742-4658.2010.07968.x

Martin, M., Colman, M. J., GomezCasati, D. F., Lamattina, L., and Zabaleta, E. J. (2009). Nitric oxide accumulation is required to protect against iron-mediated oxidative stress in frataxin-deficient Arabidopsis plants. FEBS Lett. 583, 542-548. doi: 10.1016/j.febslet.2008.12.039

Masuda, T., Suzuki, T., Shimada, H., Ohta, H., and Takamiya, K. (2003). Subcellular localization of two types of ferrochelatase in cucumber. Planta 217, 602-609. doi: 10.1007/s00425003-1019-2

Metzendorf, C., Wu, W., and Lind, M. I. (2009). Overexpression of Drosophila mitoferrin in $1(2) \mathrm{mbn}$ cells results in dysregulation of Fer1HCH expression. Biochem. J. 421, 463-471. doi: 10.1042/BJ20082231

Mochizuki, N., Tanaka, R., Grimm, B., Masuda, T., Moulin, M., Smith, A. G., et al. (2010). The cell biology of tetrapyrroles: a life and death struggle. Trends Plant Sci. 15, 488-498. doi: 10.1016/j.tplants.2010.05.012

Mukherjee, I., Campbell, N. H., Ash, J. S., and Connolly, E. L. (2006). Expression profiling of the Arabidopsis ferric chelate reductase (FRO) gene family reveals differential regulation by iron and copper. Planta 223 1178-1190. doi: 10.1007/s00425005-0165-0

Murgia, I., Delledonne, M., and Soave, C. (2002). Nitric oxide mediates iron-induced ferritin accumulation in Arabidopsis. Plant J. 30, 521528. doi: 10.1046/j.1365-313X.2002. 01312.x

Napoli, E., Morin, D., Bernhardt, R., Buckpitt, A., and Cortopassi, G. (2007). Hemin rescues adrenodoxin, heme a and cytochrome oxidase activity in frataxin-deficient oligodendroglioma cells. Biochim. Biophys. Acta 1772, 773-780. doi: 10.1016/j.bbadis.2007.04.001
Nouet, C., Motte, P., and Hanikenne, M. (2011). Chloroplastic and mitochondrial metal homeostasis. Trends Plant Sci. 16, 395-404. doi: 10.1016/j.tplants.2011.03.005

Paradkar, P. N., Zumbrennen, K. B., Paw, B. H., Ward, D. M., and Kaplan, J. (2009). Regulation of mitochondrial iron import through differential turnover of mitoferrin 1 and mitoferrin 2. Mol. Cell. Biol. 29, 1007-1016. doi: 10.1128/MCB.01 685-08

Park, S., Gakh, O., Mooney, S. M., and Isaya, G. (2002). The ferroxidase activity of yeast frataxin. J. Biol. Chem. 277, 38589-38595. doi: 10.1074/jbc.M206711200

Petrat, F., De Groot, H., and Rauen, U. (2001). Subcellular distribution of chelatable iron: a laser scanning microscopic study in isolated hepatocytes and liver endothelial cells. Biochem. J. 356, 61-69. doi: 10.1042/0264-6021:3560061

Philpott, C. C. (2012). Coming into view: eukaryotic iron chaperones and intracellular iron delivery. $J$. Biol. Chem. 287, 13518-13523. doi: 10.1074/jbc.R111.326876

Ravet, K., Touraine, B., Boucherez, J., Briat, J.-F., Gaymard, F., and Cellier, F. (2009). Ferritins control interaction between iron homeostasis and oxidative stress in Arabidopsis. Plant J. 57, 400-412. doi: 10.1111/j.1365313X.2008.03698.x

Santos, R., Lefevre, S., Sliwa, D., Seguin, A., Camadro, J. M., and Lesuisse, E. (2010). Friedreich ataxia: molecular mechanisms, redox considerations, and therapeutic opportunities. Antioxid. Redox Signal. 13, 651-690. doi: 10.1089/ars.2009.3015

Shaw, G. C., Cope, J. J., Li, L., Corson, K., Hersey, C., Ackermann, G. E., et al. (2006). Mitoferrin is essential for erythroid iron assimilation. Nature 440, 96-100. doi: 10.1038/nature 04512

Shimoni-Shor, E., Hassidim, M., YuvalNaeh, N., and Keren, N. (2010). Disruption of Nap14, a plastidlocalized non-intrinsic ABC protein in Arabidopsis thaliana results in the over-accumulation of transition metals and in aberrant chloroplast structures. Plant Cell Environ. 33, 1029-1038. doi: 10.1111/j.13653040.2010.02124.x

Tanaka, R., and Tanaka, A. (2007). Tetrapyrrole biosynthesis in higher plants. Annu. Rev. Plant Biol. 58, 321-346. doi: 10.1146/annurev.arplant.57.032905. 105448

Tarantino, D., Casagrande, F., Soave, C., and Murgia, I. (2010a). Knocking 
out of the mitochondrial AtFer4 ferritin does not alter response of Arabidopsis plants to abiotic stresses. J. Plant Physiol. 167, 453-460. doi: 10.1016/j.jplph.2009.10.015

Tarantino, D., Santo, N., Morandini, P., Casagrande, F., Braun, H. P., Heinemeyer, J., et al. (2010b). AtFer4 ferritin is a determinant of iron homeostasis in Arabidopsis thaliana heterotrophic cells. J. Plant Physiol. 167, 1598-1605. doi: 10.1016/j.jplph.2010.06.020

Turowski, V. R., Busi, M. V., and Gomez-Casati, D. F. (2012). Structural and functional studies of the mitochondrial cysteine desulfurase from Arabidopsis thaliana. Mol. Plant 5, 1001-1010. doi: 10.1093/mp/ sss037

van Lis, R., Atteia, A., Nogaj, L. A., and Beale, S. I. (2005). Subcellular localization and light-regulated expression of protoporphyrinogen IX oxidase and ferrochelatase in Chlamydomonas reinhardtii. Plant Physiol. 139, 1946-1958. doi: 10.1104/pp.105.069732

Vazzola, V., Losa, A., Soave, C., and Murgia, I. (2007). Knockout of frataxin gene causes embryo lethality in Arabidopsis. FEBS Lett. 581, 667-672. doi: 10.1016/j.febslet.2007.01.030

Victoria Fde, C., Bervald, C. M., Da Maia, L. C., De Sousa, R. O., Panaud, O., and De Oliveira, A. C. (2012). Phylogenetic relationships and selective pressure on gene families related to iron homeostasis in land plants. Genome
55, 883-900. doi: 10.1139/gen2012-0064

Vigani, G. (2012). Discovering the role of mitochondria in the iron deficiency-induced metabolic responses of plants. J. Plant Physiol. 169, 1-11. doi: 10.1016/j.jplph.2011.09.008

Vigani, G., Maffi, D., and Zocchi, G. (2009). Iron availability affects the function of mitochondria in cucumber roots. New Phytol. 182, 127-136. doi: 10.1111/j.14698137.2008.02747.x

Vigani, G., and Zocchi, G. (2009). The fate and the role of mitochondria in Fe-deficient roots of strategy I plants. Plant Signal. Behav. 4, 375-379. doi: 10.4161/psb.4.5.8344

Vigani, G., Zocchi, G., Bashir, K., Philippar, K., and Briat, J.-F. (2013). Signals from chloroplasts and mitochondria for iron homeostasis regulation. Trends Plant Sci. 18, 305-311. doi: 10.1016/j.tplants.2013.01.006

Waldherr, M., Ragnini, A., Jank, B., Teply, R., Wiesenberger, G., and Schweyen, R. (1993). A multitude of suppressors of group II intron-splicing defects in yeast. Curr. Genet. 24, 301-306. doi: 10.1007/BF00336780

Wiesenberger, G., Link, T. A., Von Ahsen, U., Waldherr, M., and Schweyen, R. J. (1991). MRS3 and MRS4, two suppressors of mtRNA splicing defects in yeast, are new members of the mitochondrial carrier family. J. Mol. Biol. 217, 23-37. doi: 10.1016/0022-2836(91)90608-9
Wu, A. C., Lesperance, L., and Bernstein, H. (2002). Screening for iron deficiency. Pediatr. Rev. 23, 171-178. doi: 10.1542/pir.23-5-171

Yadavalli, V., Jolley, C. C., Malleda, C., Thangaraj, B., Fromme, P., and Subramanyam, R. (2012). Alteration of proteins and pigments influence the function of photosystem I under iron deficiency from Chlamydomonas reinhardtii. PLoS ONE 7:e35084. doi: 10.1371/journal.pone.0035084

Yoon, T., and Cowan, J. A. (2004). Frataxin-mediated iron delivery to ferrochelatase in the final step of heme biosynthesis. J. Biol. Chem. 279, 25943-25946. doi: 10.1074/jbc.C400107200

Zancani, M., Peresson, C., Biroccio, A., Federici, G., Urbani, A., Murgia, I., et al. (2004). Evidence for the presence of ferritin in plant mitochondria. Eur. J. Biochem. 271, 3657-3664. doi: 10.1111/j.14321033.2004.04300.x

Zhang, Y., Lyver, E. R., Knight, S. A., Lesuisse, E., and Dancis, A. (2005). Frataxin and mitochondrial carrier proteins, Mrs3p and Mrs4p, cooperate in providing iron for heme synthesis. J. Biol. Chem. 280, 19794-19807. doi: 10.1074/jbc. M500397200

Zhang, Y., Lyver, E. R., Knight, S. A., Pain, D., Lesuisse, E., and Dancis, A. (2006). Mrs3p, Mrs4p, and frataxin provide iron for $\mathrm{Fe}-\mathrm{S}$ cluster synthesis in mitochondria. J. Biol. Chem. 281, 22493-22502. doi: 10.1074/jbc.M604246200
Zhao, G., Ceci, P., Ilari, A., Giangiacomo, L., Laue, T. M., Chiancone, E., etal. (2002). Iron and hydrogen peroxide detoxification properties of DNA-binding protein from starved cells. A ferritin-like DNA-binding protein of Escherichia coli. J. Biol. Chem. 277, 27689-27696. doi: 10.1074/jbc. M202094200

Conflict of Interest Statement: The authors declare that the research was conducted in the absence of any commercial or financial relationships that could be construed as a potential conflict of interest.

Received: 05 June 2013; accepted: 18 August 2013; published online: 06 September 2013.

Citation: Jain A and Connolly EL (2013)

Mitochondrial iron transport and homeostasis in plants. Front. Plant Sci. 4:348. doi: 10.3389/fpls.2013.00348

This article was submitted to Plant Nutrition, a section of the journal Frontiers in Plant Science.

Copyright (c) 2013 Jain and Connolly. This is an open-access article distributed under the terms of the Creative Commons Attribution License (CC BY). The use, distribution or reproduction in other forums is permitted, provided the original author(s) or licensor are credited and that the original publication in this journal is cited, in accordance with accepted academic practice. No use, distribution or reproduction is permitted which does not comply with these terms. 